\title{
RANDOM VORTEX METHOD IN NUMERICAL ANALYSIS OF 2D FLOW AROUND CIRCULAR CYLINDER
}

\author{
STANISŁAW KOSTECKI \\ Wrocław University of Technology, Wrocław, Poland, \\ e-mail: stanislaw.kostecki@pwr.edu.pl
}

\begin{abstract}
A combination of the vortex method and the boundary element method is used here to predict the two-dimensional flow field around a circular cylinder. Cylindrical structures experience strong hydrodynamic loading, due to vortex detachment from the both sides of cylinder during the flow. Thus, the practical meaning of such calculation is significant particularly in offshore oil and gas engineering as well as in the bridge and hydraulic structure engineering. This paper presents the mathematical formulation of the vortex method for the velocity and vorticity field calculation. The calculated velocity and vorticity fields are then used to predict the pressure distribution on the cylinder surface by the boundary element method. The resulting pressure on the cylinder, the Strouhal number and the length of the base recirculation zone are compared with solutions of other numerical methods and experiments, and a good agreement is achieved.
\end{abstract}

Key words: vorticity, vortex method, flow instability, circular cylinder, boundary element method

\section{NOTATIONS}

$\alpha$ - an exterior angle in BEM

$\alpha=\left[\alpha_{1}, \alpha_{2}\right]-$ initial position of vortex particle

$d s_{i}-$ length of vorticity layer segment

$\nabla^{2}$ - Laplace operator

$\varepsilon$ - mollifying function scaling parameter - cut radius

$f_{\varepsilon}(\mathbf{x})$ - mollifying (cut) function

$\gamma$ - vortex sheet intensity

$\Gamma(t)$ - vortex circulation

$\eta=\left(\eta_{1}, \eta_{2}\right)-$ random particle displacement

$k t$ - number of time step

$\mathbf{K}(\mathbf{x}), G(\mathbf{x})$ - kernels of Biot-Savart formula

$\mathbf{K}_{\varepsilon}(\mathbf{x}), G_{\varepsilon}(\mathbf{x})$ smoothed kernels of Biot-Savart formula

$f$ - frequency of vortex separation

$\hat{\mathbf{n}}$ - unit vector normal to boundary

$N$ - vortex number

$v$ - kinematic viscosity coefficient

$p$ - fluid's pressure

$\mathrm{Re}$ - Reynolds number

$\mathrm{Sh}-$ Strouhal number

$t$ - time

$\hat{\mathbf{t}}-$ unit vector tangent to boundary

$\Delta t$ - time step

$\mathbf{u}=\left(u_{1}, u_{2}\right)-$ velocity vector

$\mathbf{u}_{\omega}$ - non-divergent component of velocity field

$\mathbf{u}_{p}$ - potential component of velocity field

$\mathbf{u}_{\infty}-$ velocity on outer boundaries of flow area

$\omega$ - vorticity

$\psi$ - stream function

$\mathbf{X}(\alpha, t)-$ particle trajectory

\section{INTRODUCTION}

The turbulence flow past a circular cylinder causes separation of flow and occurrence of vortex shedding consists of alternate rotating vortices shed periodically from both sides of the cylinder. Instability of the flow around the cylinder causes periodic variable hydrodynamic loads on the cylinder, which can lead to noise and vibration of the structure. These phenomena have been widely investigated because of the complexities and practical importance, especially in the offshore oil and gas engineering as well as the bridge and hydraulic structure engineering.

The numerical analysis of the characteristic curve of the cylinder flow around may be based on various methods. The most popular ones include the grid methods, e.g., FEM, FDM, FVM, and the methods based on the particle movement, such as the vortex method and, lately, the Bolzman Latice (Fallah [3]). By these methods, various turbulent flow models are solved. The most frequently used include RANS, that consists in solving the Reynolds equation set (averaged Navier and Stokes equations), closed with additional equations connecting the turbulent viscosity with the kinetic energy of the turbulence and its dissipation $(k-\varepsilon)$ or the energy and the vorticity of the flow field $(k-\omega)$. This last model, in connection with 
FVM, for investigation of cylinder flow around for various Reynolds numbers, was applied, among others, by Stringer [16] to determine the development of the vortex length, pressure and drag coefficient versus Reynolds number, and Guilmineau and Queutey [6] used RANS to study the dynamics and fluid forcing on an elastically mounted rigid cylinder oscillating transversely to a free stream.

Since the 1980's, a fast development of the LES method has been observed, that was proposed in 1963 by Smagorinsky, differing from RANS in that the flow field is averaged spatially, and not upon time. There are determined the vortices of the dimensions comparable with the net mesh dimensions, while the lower vortices are taken into consideration by introducing an extra viscosity following from the stresses in the lower scale vortices (Subgrid Scale Stress).

Al-Jamal and Dalton [1] used LES modeling in performing the analysis of the behavior of drag and inertia coefficients in 2D flow over a cylinder under conditions of currents inline with waves for two Reynolds numbers $-15,000$ and 500. Catalano et al. [2] presented the comparison of flow around a cylinder by LES and RANS.

Among the grid-free methods, the most popular in the cylinder flow around problem is the vortex particle simulation. Shademan and Nouri [14] analyze the trajectory of a bubble in two-phase bubby flow by the Random Vortex Method for Re 3000. Huang and Wu [8] investigated numerically particle-laden water flows past a circular cylinder by the discrete vortex method to evaluate trajectories of solid particles in the unsteady water flow field. The flow past the tandem of cylinders was tested, using deterministic vortex method, by, among others, Huang et al. [7]. Liang et al. [12] used RVM for analyzing drag and lift forces on two-dimensional cylinder beneath a rigid wall and a free surface.

This paper presents the random vortex method (RVM) in analysis of the flow around 2D circular cylinder for the high Reynolds number. RVM is the approach used in determining velocity and vorticity fields in the proximity of the cylinder and the boundary element method (BEM) to calculate the pressure. The results are compared with the experiment presented by Zdravkovich [17] and the numerical calculations by RANS and LES presented by Catalano et al. [2].

The remainder of the paper is organized as follows. The next section consists of a brief description of the mathematical basis and the discrete vortex method formulation. Then the solution of pressure, filed by boundary element method, is presented. Finally, the calculations of the mean pressure coefficient on cylin- der are provided and the base pressure coefficient, the Strouhal number and recirculation zone length are discussed and compared to experiments and calculation done by another numerical method.

\section{MATHEMATICAL BASIS}

Let us consider the case of a 2D flow of an incompressible viscous fluid in area $D$, described by the Helmholtz equation in terms of the velocity and vorticity

$$
\frac{\partial \omega}{\partial t}+\mathbf{u} \cdot \nabla \omega-v \nabla^{2} \omega=0, \quad \mathbf{x}=\left(x_{1}, x_{2}\right) \in D, \quad t>0,
$$

where $\mathbf{u}(\mathbf{x}, t)=\left[u_{1}(\mathbf{x}, t), u_{2}(\mathbf{x}, t)\right]$ is the velocity vector, $\omega=\partial u_{2} / \partial x_{1}-\partial u_{1} / \partial x_{2}-$ the vorticity scalar ${ }^{1}, v-$ the coefficient of kinematic viscosity.

Equation (1) must be completed with the one of the mass conservation (2) and the initial condition (3)

$$
\begin{gathered}
\nabla \cdot \mathbf{u}=\frac{\partial u_{1}}{\partial x_{1}}+\frac{\partial u_{2}}{\partial x_{2}}=0, \\
\left.\omega\right|_{t=0}=\omega_{0}
\end{gathered}
$$

and the no-through-flow and no-slip-flow conditions on the solid boundary $S$.

Vorticity is equal to the curl of its velocity field $\mathbf{u}$

$$
\omega=\nabla \times \mathbf{u}
$$

and due to (3) the curl of vorticity field is given as

$$
\nabla \times \omega=\nabla \times(\nabla \times \mathbf{u})=\nabla(\nabla \cdot \mathbf{u})-\nabla \mathbf{u}=-\nabla^{2} \mathbf{u} .
$$

\section{FUNDAMENTALS OF DISCRETE RANDOM VORTEX METHOD}

The advantage of the vortex method is that the calculations of flow for an arbitrary Reynolds number are grid free and without the use of any RANS type turbulent models. The vortex method consists in substituting of the continuous vorticity field by a set of $N$ regularized vortex particles that move along the trajectories $\mathbf{X}\left(\alpha_{j}, t\right)$ in accordance with the momentary velocity field

$$
\omega(\mathbf{x}, t)=\sum_{j=1}^{N} f_{\varepsilon}\left(\mathbf{x}-\mathbf{X}\left(\boldsymbol{\alpha}_{j}, t\right)\right) \Gamma_{j},\left.\quad \mathbf{X}\left(\boldsymbol{\alpha}_{j}, t\right)\right|_{t=0}=\boldsymbol{\alpha}_{j},
$$

\footnotetext{
${ }^{1}$ Actually, the vorticity in the $2 \mathrm{D}$ flow is a vector, but directed vertically to the flow plane.
} 
where $\mathbf{X}\left(\boldsymbol{\alpha}_{j}, t\right)$ is the position of the $j$-th vortex particle in time $t, \alpha_{j}=\left(\alpha_{1}^{j}, \alpha_{2}^{j}\right)$ is the onset position of the $j$-th particle, $\Gamma_{j}$ is the vortex particle strength and $f_{\varepsilon}(\mathbf{x})$ is the standard mollifying function causing that the vortex particles have a limited support. If the fluid velocity field is properly smooth, then the trajectory of particle is given by the relation

$$
\frac{d \mathbf{X}\left(\boldsymbol{\alpha}_{j}, t\right)}{d t}=\mathbf{u}\left(\mathbf{X}\left(\boldsymbol{\alpha}_{j}, t\right), t\right) .
$$

Knowing the vorticity field, one may calculate the equivalent velocity field from relation (5). Its solution follows from the generalized Biot-Savart law

$$
\mathbf{u}(\mathbf{x}, t)=\sum_{j=1}^{N} \mathbf{K}_{\varepsilon}\left(\mathbf{x}-\mathbf{X}\left(\boldsymbol{\alpha}_{j}, t\right)\right) \Gamma_{j},
$$

where the vector function $\mathbf{K}_{\varepsilon}$ is the convolution of kernel $\mathbf{K}$ with the mollifying function $f_{\varepsilon}$

$$
\mathbf{K}_{\varepsilon}=\mathbf{K}_{\varepsilon} * f_{\varepsilon} .
$$

The kernel function for a flat flow has the following form

$$
\mathbf{K}(\mathbf{x})=\frac{\left(-x_{2}, x_{1}\right)}{2 \pi|\mathbf{x}|^{2}}
$$

and, as the mollifying function, we use the Cauchy function in the following form

$$
f_{\varepsilon}(\mathbf{x})=\frac{1}{\pi \varepsilon}\left(1+\left|\frac{\mathbf{x}}{\varepsilon}\right|^{2}\right)^{-2}, \quad \varepsilon>0,
$$

which is smooth and axially symmetric, with the carrier rapidly decreasing to zero in infinity, and fulfilling specific conditions ensuring the convergence and stability of the method (Majda and Bertozzi [13]).

The essence of the random vortex method is the decomposition of the vorticity transport equation (1) into the convection current equation

$$
\frac{\partial \omega}{\partial t}+\mathbf{u} \cdot \nabla \omega=\frac{D \omega}{D t}=0
$$

and the diffuse equation

$$
\frac{\partial \omega}{d t}=v \nabla^{2} \omega
$$

From (12), one can conclude that vorticity of the particle is prevented during the time

$$
\omega(\mathbf{X}(\boldsymbol{\alpha}, t), t)=\omega_{0}(\boldsymbol{\alpha}) .
$$

The fundamental solution of the heat diffusion equation (13) is the Green function which is in accordance with a probability density function with mean deviation of zero and variance $2 t v$ (Lewis [11]), (Kostecki [9])

$$
\mathbf{G}(\mathbf{x}, t)=\frac{1}{\sqrt{4 \pi v t}} \exp \left(-\mathbf{x}^{2} / 4 \pi v\right) .
$$

This means that the particles are subjected to random displacement

$$
\boldsymbol{\eta}\left(\boldsymbol{\alpha}_{j}, t\right) ; \boldsymbol{\eta}=\left(\eta_{x 1}, \eta_{x 2}\right) .
$$

which depends on the viscosity and the time step.

The calculation consists in determining the evolution of vorticity field (approximated by the set of vortex particles) in successive time steps $\Delta t$. In each time step, the vortex particles are moved due to advection in accordance with equation (7) and, next, each particle is subject to a random shift in conformity with equation (16)

$$
\mathbf{X}\left(\boldsymbol{\alpha}_{j}, t+\Delta t\right)=\mathbf{X}\left(\boldsymbol{\alpha}_{j}, t\right)+\Delta t \mathbf{u}_{j}(t)+\eta\left(\boldsymbol{\alpha}_{j}, t\right)
$$

where the velocity vector $\mathbf{u}$ is calculated on the basis of equation (8).

\section{BOUNDARY CONDITIONS}

To satisfy the no-through-flow boundary condition, the velocity field $\mathbf{u}(\mathbf{x}, t)$ is modified by adding a potential velocity field $\mathbf{u}_{p}(\mathbf{x}, t)$ such that the normal component $\left(\mathbf{u}_{p}+\mathbf{u}\right) \cdot \hat{\mathbf{n}}=0$ on the boundary of the cylinder. It can be proved that an additional potential field will have no effect on the vortex evolution solution (Kostecki [10]).

In our calculation, the boundary element method is used to determine the potential velocity field $\mathbf{u}_{p}$ from the gradient of the potential stream function $\psi_{p}$ which satisfies the Laplace equation

$$
\nabla^{2} \psi_{p}=0
$$

with the boundary conditions for the stream function $\psi_{p}=-\psi_{\omega}$ (Fig. 2). The stream function $\psi_{\omega}$ can be approximated as follows

$$
\begin{gathered}
\psi_{\omega}(\mathbf{x}, t)=\sum_{j} G_{\varepsilon}\left(\mathbf{x}-\mathbf{X}\left(\boldsymbol{\alpha}_{j}, t\right) \Gamma_{j},\right. \\
G_{\varepsilon}=G * f_{\varepsilon}, \quad G(\mathbf{x})=-(2 \pi)^{-1} \ln |\mathbf{x}| .
\end{gathered}
$$

To satisfy the no-slip-flow boundary conditions on the cylinder's wall, the process of vorticity generating 
by nascent vortex sheet $\gamma(\mathbf{x}, \mathrm{t})$ is introduced. The intensity of the vortex sheet can be determined by solving the 2nd kind Fredholm integral equation derived by the author in (Kostecki [10])

$$
\begin{gathered}
\gamma(\xi)=-2\left(\mathbf{u}+\mathbf{u}_{p}\right)(\xi) \cdot \hat{\mathbf{t}}(\xi) \\
+\frac{1}{\pi} \int_{S} \gamma(\mathbf{x}) \frac{\partial \ln |\mathbf{x}-\xi|}{\partial \hat{\mathbf{n}}(\xi)} d S(\mathbf{x}), \quad \mathbf{x}, \xi \in S,
\end{gathered}
$$

where $\hat{\mathbf{n}}(\boldsymbol{\xi})$ and $\hat{\mathbf{t}}(\boldsymbol{\xi})$ are the unit vectors normal directed outwards and tangent to the cylinder at a point $\xi$, respectively. After its generation, the vortex sheet is discretized to vortex particles whose strength is given by

$$
\Gamma_{i}=-\int_{d s_{i}} \gamma(\mathbf{x}) \cdot d S(\mathbf{x}), \quad \mathbf{x} \in S
$$

where $d s_{i}$ stands for the elementary segments into which the boundary of the cylinder is divided. As a result, in each time step "new" vortex particles are generated in the centres of the segments, compensating the tangent velocity component on the solid boundary.

\section{PRESSURE AND HYDRODYNAMIC FORCE}

From the point of view of engineering practice, pressure field distribution is one of the most important characteristics of the flow. The RVM method allows one to determine the velocity and vortex field distributions from which the pressure in the flow can be calculated. There are two approaches commonly used for this purpose. One approach is to integrate the $N-S$ equations to determine the pressure gradient. But then unavoidable numerical errors appear in the solution. The other approach consists in solving the Poisson equation for pressure by applying divergence to the $N-S$ equations (Gresho [5])

$$
-\nabla^{2} P=\operatorname{div} \mathbf{B} \text { in } D
$$

with the Neumann boundary condition

$$
\frac{\partial P}{\partial n}=-\mathbf{B} \cdot \hat{\mathbf{n}} \text { on } S,
$$

where $P=p / \rho$ is the kinematic pressure, $\mathbf{B}=\frac{\partial \mathbf{u}}{\partial t}$ $\left.+(\mathbf{u} \nabla \mathbf{u})-v \nabla^{2} \mathbf{u}\right), \hat{\mathbf{n}}$ is a vector normal to boundary $S$ and directed outwards bounded area $D$, in which the flow is considered.

The boundary element method (BEM), which allows one to write relation (15) in the integral form, was used to solve the Poisson problem. After appropriate transformations (Kostecki [9]) the equation assumed the form which made it possible to determine pressure at any point $\xi$ on the boundary

$$
\begin{gathered}
c(\xi) P(\xi)+\int_{S} P \frac{\partial P^{*}(\mathbf{x}, \boldsymbol{\xi})}{\partial \hat{\mathbf{n}}} d S(\mathbf{x}) \\
=-\int_{D} \mathbf{B} \cdot \nabla P^{*}(\mathbf{x}, \boldsymbol{\xi}) d \mathbf{x},
\end{gathered}
$$

where $P^{*}(\mathbf{x}, \xi)=-1 / 2 \pi \ln |\mathbf{x}|$ (Gaul et al. [4]) and $c(\xi)=1-\alpha / 2 \pi$ where $\alpha$ is an exterior angle formed at point $\xi$ by the tangents to the boundary. The advantage of this approach is that the right side of the equation depends on the value of vector $\mathbf{B}$ only inside area $D$, and not on the boundary where $\mathbf{B}$ could be singular.

Solution (24) requires that vector function $\mathbf{B}$ be integrated over the whole area $D$. Having in mind the effectiveness and accuracy of the numerical calculations, the integrals were transformed to forms containing expressions with only first-order derivatives

$$
\begin{gathered}
\int_{D} \mathbf{B} \cdot \nabla P^{*}(\mathbf{x}, \boldsymbol{\xi}) d \mathbf{x}=\int_{S} P^{*}(\mathbf{x}, \boldsymbol{\xi}) \frac{\partial \mathbf{u}}{\partial t} \cdot \hat{\mathbf{n}} d S(\mathbf{x}) \\
+\int_{D} \nabla\left(\frac{\mathbf{u}^{2}}{2}\right) \cdot \nabla_{\mathbf{x}} P^{*}(\mathbf{x}, \boldsymbol{\xi}) d \mathbf{x}+\int_{D} \omega\left(\mathbf{u} \times \nabla_{\mathbf{x}} P^{*}(\mathbf{x}, \boldsymbol{\xi})\right) d \mathbf{x} \\
-v \int_{D}(\nabla \times \boldsymbol{\omega}) \cdot \nabla_{\mathbf{x}} P^{*}(\mathbf{x}, \boldsymbol{\xi}) d \mathbf{x} .
\end{gathered}
$$

The velocity $\mathbf{u}(\mathbf{x}, t)$ functions and the vorticity functions $\omega(\mathbf{x}, t)$ in equation (18) are previously determined by RVM; $\nabla_{\mathbf{x}}$ stands for a gradient relative to the variable $\mathbf{x}$.

Flow area $D$ was divided into triangular elements and integration over the area was replaced by the sum of the integrals over the elements. The integrals were calculated approximately using numerical quadrature. For a detailed description of the above transformations and the solution, see (Kostecki [9]).

\section{FLOW AROUND CYLINDER}

The cylinder under investigation is located in a free stream flow. Aassuming $D=1.0$ and velocity $\mathbf{u}_{\infty}=[1,0]$ 
as the characteristic quantities, the non-dimensional calculations were done for the Reynolds number: $\operatorname{Re}=10^{6}$, that constitutes the border between the critical flow regime and the super-critical border regime, in conformity with the division proposed by Zdravkovich [18]. The cylinder circumference was replaced by 128 segments with the non-slip-flow condition on each of them. For the purposes of the numerical calculations, the flow area was limited to a rectangle of dimensions $20.0 \times 10.0$; the location of the cylinder center was assumed at a distance of 4.0 from the inlet and in the medium of the rectangle height. At the initial instant, $t=0$, the velocity field is potential, uniform, of the value $\left.\mathbf{u}\right|_{t=0}=[1,0]$ within the whole area. The scheme of the calculation domain is shown in Fig. 1.

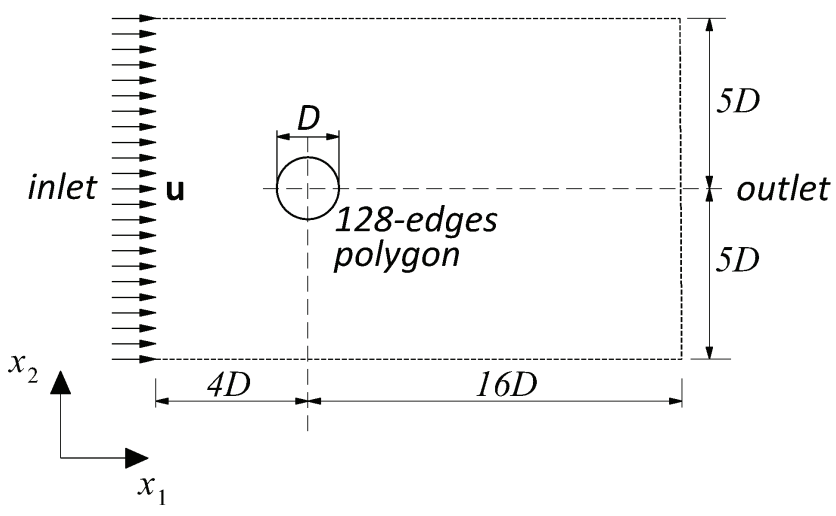

Fig. 1. Scheme of the flow area

The calculations were carried out assuming the time step of $\Delta t=0.1$, the maximum number of steps amounted to 2000, the values of the current functions were determined at numerical grid vortices, of dimensions $0.4 \times 0.2$, the stream functions averaged upon time were determined after each time step.

The numerical simulation performed shows that vortices form a characteristic path, consisting of positive and negative circulation vortex structures, behind the cylinder (Fig. 2). The path grows downstream due to vorticity diffusion and relative width $H / L$ stands at about 0.3 , which is in good agreement with the experimental value (Zdravkovich [17]), where $H$ is the distance between the rows of vortices and $L$ stands for the wavelength between the same sign of vortices.

The vortices detached from both sides of the cylinder cause quasi-periodical changes in pressure. This constitutes the hydrodynamic lift and drag forces with a frequency that can be characterized by a non-dimensional Strouhal number

$$
\mathrm{Sh}=\frac{f D}{u_{\infty}}
$$

where $f$ is the frequency of the vortex shedding from the cylinder.

Figure 3 shows the instantaneous reduced vorticity circulation at the point of $x=5.5$ and $y=5.0$, i.e., $1 D$ behind the cylinder. The mean value of the circulation is 0.0 and its standard deviation amounts to 3.15. The frequency of the vortex shedding obtained by applying FFT to the vorticity signal (in the time interval from the 1000th to the 2000th time steps) is 0.22 (Fig. 3) which is very close to the experimental data presented in Table 1.

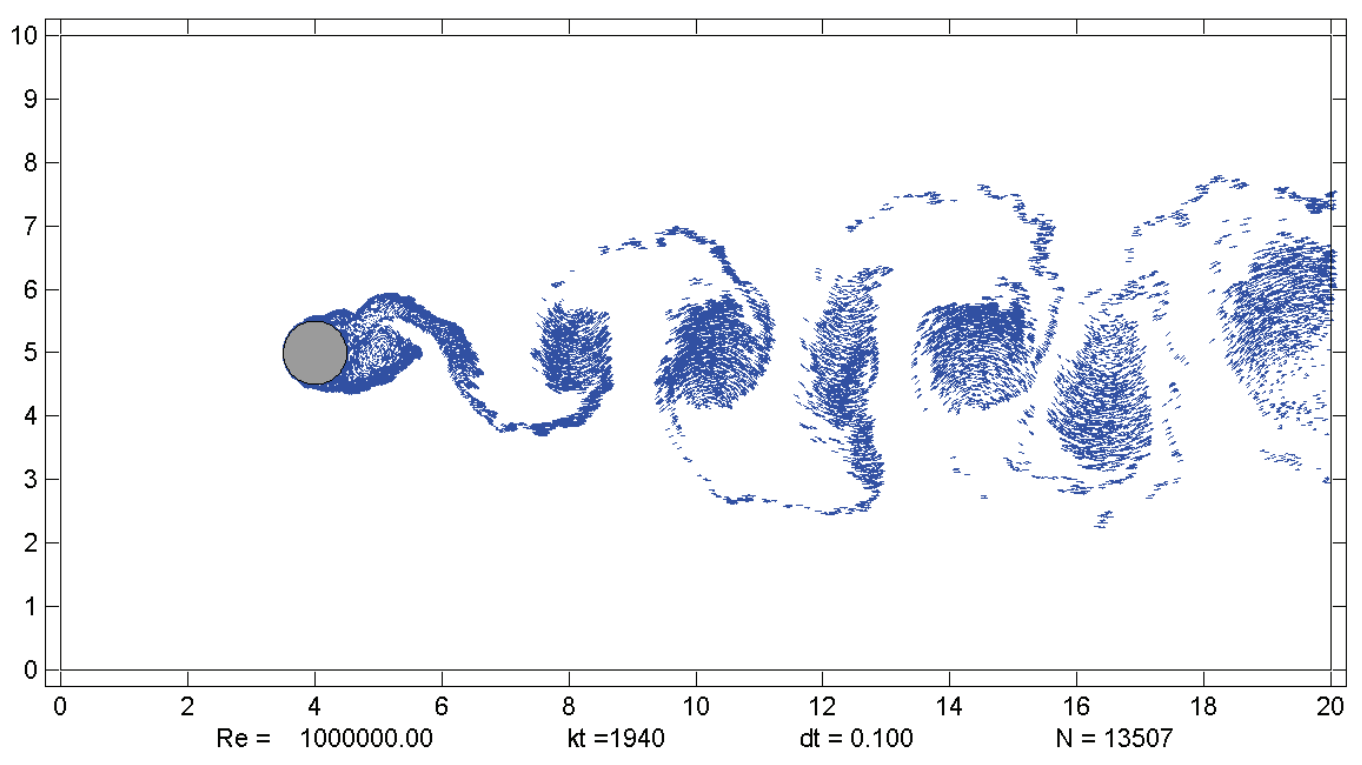

Fig. 2. Vortex path behind cylinder at $\mathrm{Re}=10^{6}$ 

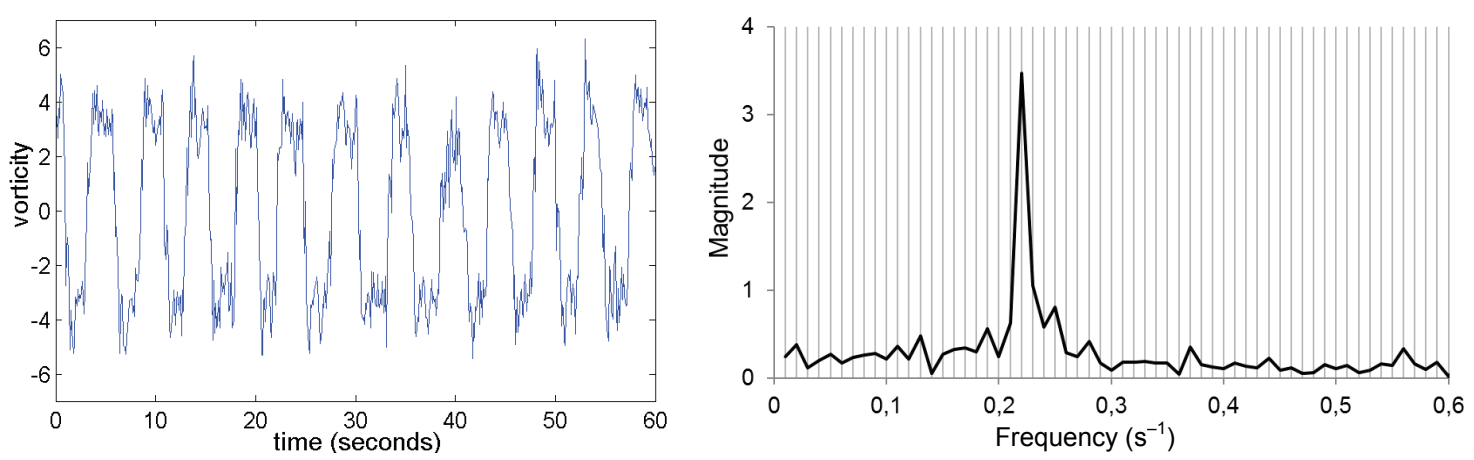

Fig. 3. Time history of vorticity circulation (left) and power spectrum (right)

Eddy formed behind the cylinder in its immediate vicinity is called the main recirculation zone. Its length is an important parameter and has been obtained by calculating the stream function and averaging it over 200 time steps. In Fig. 4, the length measurement of the recirculation zone is presented.

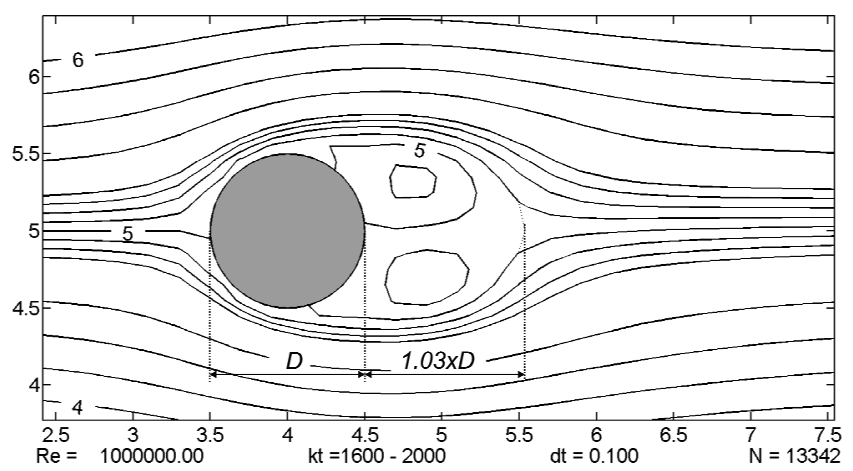

Fig. 4. Length of the recirculation zone obtained for the uniform flow at $\operatorname{Re}=10^{6}$

The most important parameter of the flow around a cylinder is the pressure value on its circumference, on the basis of which one may determine the hydrodynamic lift and drag forces. The pressure value depends, significantly, on the Reynolds number. To enable comparison of the calculated pressure values, its dimensionless form called the pressure coefficient, $C_{p}$, is usually applied

$$
C_{p}=\frac{2 p}{\rho\left|\mathbf{u}_{\infty}\right|^{2}} .
$$

In the present paper, the distribution of the pressure coefficient on the circumference of the cylinder has been calculated by DRVM + BEM and compared with results of simulation of the RANS and LES methods reported by Catalano et al. [2], as well as the laboratory tests taken by Falchsbart (in Zdravkovich [17]) (Fig. 4).

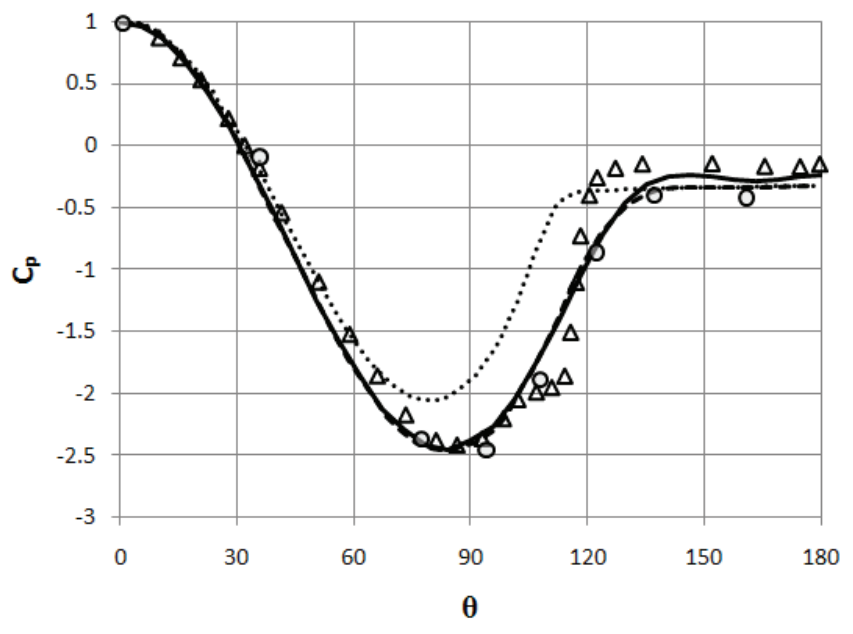

Fig. 5. Mean pressure coefficient on the cylinder circumference: $(-) \mathrm{RVM}+\mathrm{BEM}$ at $\mathrm{Re}=1 \times 10^{6} ;(-\cdot-) \mathrm{LES}$ at $\mathrm{Re}=1 \times 10^{6}$ Catalano et al. [2]; ( $\cdots)$ RANS at $\mathrm{Re}=1 \times 10^{6}$ Catalano et al. [2]; (०) experiment by Warschauer and Leene (in Zdravkovich [17]) at $\operatorname{Re}=1.2 \times 10^{6} ;(\triangle)$ experiment by Falchsbart

(in Zdravkovich [17]) at $\operatorname{Re}=6.7 \times 10^{5}$

The calculated pressure coefficient $C_{p}$ behind the cylinder, recirculation zone length $L_{r}$ and vortex structure formation frequency expressed by the Strouhal number are compared with the ones determined by LES, RANS and the experiments in Table 1.

Table 1. Base pressure coefficient, Strouhal number and recirculation zone length for flow around cylinder

\begin{tabular}{|l|c|c|c|c|}
\hline & $\mathrm{Re}$ & Base $C_{p}$ & $L_{r}$ & Sh \\
\hline RVM+BEM & $10^{6}$ & -0.29 & 1.03 & 0.21 \\
\hline LES Catalano et al. [2] & $10^{6}$ & -0.32 & 1.04 & 0.35 \\
\hline RANS Catalano et al. [2] & $10^{6}$ & -0.33 & - & 0.33 \\
\hline RVM Liang et al. [12] & $4 \times 10^{5}$ & - & - & 0.21 \\
\hline Exp. Shih et al. [15] & $10^{6}$ & -0.33 & - & 0.22 \\
\hline $\begin{array}{l}\text { Exp. Falchsbart } \\
\text { (in Zdravkovich [17]) }\end{array}$ & $6.7 \times 10^{5}$ & -0.17 & - & $0.18-0.50$ \\
\hline
\end{tabular}

As follows from Table 1, the results of the flow simulation around the cylinder are comparable with 
those of the other numerical methods and with the experiments. The obtained Strouhal number value is consistent with that of the experiment, but differs from the calculations by the LES and RANS methods, which may be a result of the assumption of the smooth boundary accepted by Catalano et al. [2] for the cylinder surface.

An improvement of the calculation accuracy for the vortex method may be achieved by assuming a smaller time step and more dense division of the cylinder edge; however, it will result in an increase of the vortex number and in the increased calculation time. Current results were obtained for a relatively low number of vortices - about 14000 and a relatively great non-dimensional time step $\mathbf{u}_{\infty} d t / D_{c}=0.1$. For the compared calculations by the LES method (Catalano et al. [2]), the time step was 0.003 .

\section{CONCLUSIONS}

Combining the Random Vortex and Boundary Element Methods is developed using in-house code to simulate two-dimensional flow around circular cylinder. RVM is used for solving the velocity and vorticity fields. An advantage of RVM versus RANS or LES is mesh free-self adaptive algorithm which solves the turbulence flow directly without the need to use turbulence closure models. The BEM is used to calculate the pressure field. The advantage of this approach is that the solution of pressure depends on the value of velocity and vorticity inside the area of the flow and does not depend on the values on the boundaries where they could be infinite. The averaged values of flow parameters, i.e., the pressure distribution on the cylinder circumference, the length of the recirculation zone and the Strouhal number are calculated. A very good agreement between the calculation results and the experimental data and the RANS/LES calculation results confirms the validity of the current method.

The proposed method, being a combination of RVM and BEM, allows one to calculate, with a high degree of accuracy, the pressure, and in consequence the hydrodynamic forces acting on the cylindrical structures in a stream flow.

\section{REFERENCES}

[1] Al-Jamal H., Dalton C., Two-dimensional numerical simulation of a wave with a current past a circular cylinder. Part 1. Inline flow, Applied Mathematical Modelling, 2013, Vol. 37, 7521-7538.
[2] Catalano P., Wang M., Iaccarino G., Moin P., Numerical simulation of the flow around a circular cylinder at high Reynolds numbers, Int. J. of Heat and Fluid Flow, 2003, Vol. 24, 463-469.

[3] Fallah K., Fardad A., Fattahi E., Sedaghati Zadeh N., GHADERI A., Numerical simulation of planar shear flow passing a rotating cylinder at low Reynolds numbers, Acta Mech., 2012, Vol. 223, 221-236.

[4] Gaul L., KöGl M., Wagber M., Boundary element methods for engineers and scientists, an introductory course with advanced topics, Springer-Verlag, Berlin-Heidelberg 2003.

[5] Gresho P.M., On Pressure Boundary Conditions for the Incompressible Navier-Stokes Equations, Int. J. Num. Meth. Fluids, 1987, Vol. 7, 1111-1145.

[6] Guilmineau E., Queutey P., Numerical simulation of vortex-induced vibration of a circular cylinder with low massdamping in a turbulent flow, Journal of Fluids and Structures, 2004, Vol. 19, 449-466.

[7] Huang G., Huan H., Xu X., LiU Y., Simulation of Flow Past Two Tandem Cylinders Using Deterministic Vortex Method, Thermal Science, 2012, Vol. 16, No. 5, 1460-1464.

[8] Huang Y., Wu W., Numerical Study Of Particle Distribution In Wake Of Liquid-Particle Flows Past A Circular Cylinder Using Discrete Vortex Method, Appl. Math. Mech., 2006, Vol. 27(4), 535-542.

[9] Kostecki S., Numerical Determination of the Hydrodynamic Pressure Acting on a Hydraulic Gate, Polish J. of Environ. Stud., 2007, Vol. 16, No. 6B, 39-45.

[10] Kostecki S., Numerical modelling of flow through moving water-control gates by vortex method. Pt. I. Problem formulation, Archives of Civil and Mechanical Engineering, 2008, Vol. VIII, No. 3, 73-89.

[11] LewIS R.I., Vortex Element Methods for Fluid Dynamic Analysis of Engineering Systems, Cambridge University Press, London, 2005.

[12] Liang H., Zonga Z., Zoub L., Zhoua L., Suna L., Vortex shedding from a two-dimensional cylinder beneath a rigid wall and a free surface according to the discrete vortex method, European Journal of Mechanics B/Fluids, 2014, Vol. 43, 110-119.

[13] Majda A.J., Bertozzi A.L., Vorticity and Incompressible Flow, Cambridge University Press, Cambridge 2002.

[14] Shademan M., Nouri M., A Lagrangian-Lagrangian Model for Two-Phase Bubbly Flow Around Circular Cylinder, Journal of Computational Multiphase Flows, 2014, Vol. 6, No. 2, 151-168.

[15] Shin W.C.L., Wang C., Coles D., Roshko A., Experiments on flow past rough circular cylinders at large Reynolds numbers, Journal of Wind Engineering and Industrial Aerodynamics, 1993, Vol. 49, Iss. 1-3, 351-368.

[16] Stringer R.M., ZANG J., Hillis A.J., Unsteady RANS computations of flow around a circular cylinder for a wide range of Reynolds numbers, Ocean Engineering, 2014, Vol. 87, 1-9.

[17] Zdravkovich M.M., Flow Around Circular Cylinders, Vol. 1. Fundamentals, Oxford Scientific, Oxford 1997.

[18] ZdRavkovich M.M., Conceptual overview of laminar and turbulent flows past smooth and rough circular-cylinders, J. Wind Eng. Ind. Aerodyn., 1990, Vol. 33, 53-62. 